\title{
Design of New Launch and Interferometer Systems for the IMGC-02 Absolute Gravimeter
}

\author{
Andrea Prato, Sergio Desogus, Claudio Origlia, Marco Bisi, \\ and Alessandro Germak
}

\begin{abstract}
For the measurement of the acceleration due to gravity, INRiM developed a transportable ballistic rise-and-fall absolute gravimeter, the IMGC-02. It uses laser interferometry to measure the symmetrical free rising and falling motion of a test mass in the gravity field. The launch system is composed of a moveable carriage fixed to two pairs of springs loaded by an electric stepper motor, which vertically throw up a corner cube retroreflector in vacuum. The interferometer system is a modified Mach-Zehnder interferometer where the launched corner cube acts as the reflector in one of the optical arms of the interferometer and the other retroreflector acts as inertial reference during the measurement. However, both systems entail some practical problems and uncertainty contributions that need to be reduced. In particular, the current launch system might cause beam shear and rotational effects due to unavoidable small different loadings of the springs, while the current interferometer system poses problems in the alignment of the mirrors, which is a highly time-consuming procedure and has to be performed before and, sometimes, during the measurement session. For this reason, a new launch system consisting of an electric linear motor which produces a linear force along its length, and a modified Jamin interferometer system entailing a simpler alignment and a better stability in time, have been designed. This works deals with the description of these new systems.
\end{abstract}

\section{Keywords}

Absolute gravimeter $\cdot$ Gravimetry $\cdot$ Interferometer $\cdot$ Rise-and-fall gravimeter

\section{Introduction}

Absolute measurements of the acceleration due to gravity, $g$, are performed by absolute gravimeters, traceable to the units of length and time through their primary standards. For this purpose, INRiM developed a transportable ballistic rise-andfall absolute gravimeter, the IMGC-02, which is recognized as the Italian primary standard because of the WGG/04-

A. Prato $(\bowtie) \cdot$ S. Desogus $\cdot$ C. Origlia $\cdot$ M. Bisi $\cdot$ A. Germak INRiM - Istituto Nazionale di Ricerca Metrologica, Torino, Italy e-mail: a.prato@inrim.it
41 resolution $^{1}$ and, as such, is listed in the BIPM KCDB. ${ }^{2}$ The measurement of $g$ is performed using the reconstructed rise and fall motion of a corner cube retroreflector, which moves vertically in vacuum. An interferometer system is implemented in order to obtain time and space coordinates of the trajectory using a visible laser beam. The interferometer measures the distance between a free-falling corner

\footnotetext{
${ }^{1}$ Resolution WGG/04-41 of the first joint meeting of the CCM WGG and SGCAG (26-27 May 2004, BIPM): the first joint meeting of the CCM WGG and SGCAG recognized the absolute ballistic method of measurement of the acceleration due to gravity as a primary method.

${ }^{2}$ https://www.bipm.org $/ \mathrm{kcdb} / \mathrm{cmc} / \mathrm{search}$ ?domain=PHYSICS\&areaId= $4 \&$ keywords=italy \&specificPart.branch $=19 \&$ specificPart.service $=$ $41 \&$ specificPart.subService $=124 \&$ specificPart.individualService $=$ $404 \&$ _countries $=1 \&$ publicDateFrom $=\&$ publicDateTo $=\&$ unit $=$ $\& \min$ Value $=\& \max$ Value $=\& \operatorname{minUncertainty}=\& \max$ Uncertainty $=$.
} 

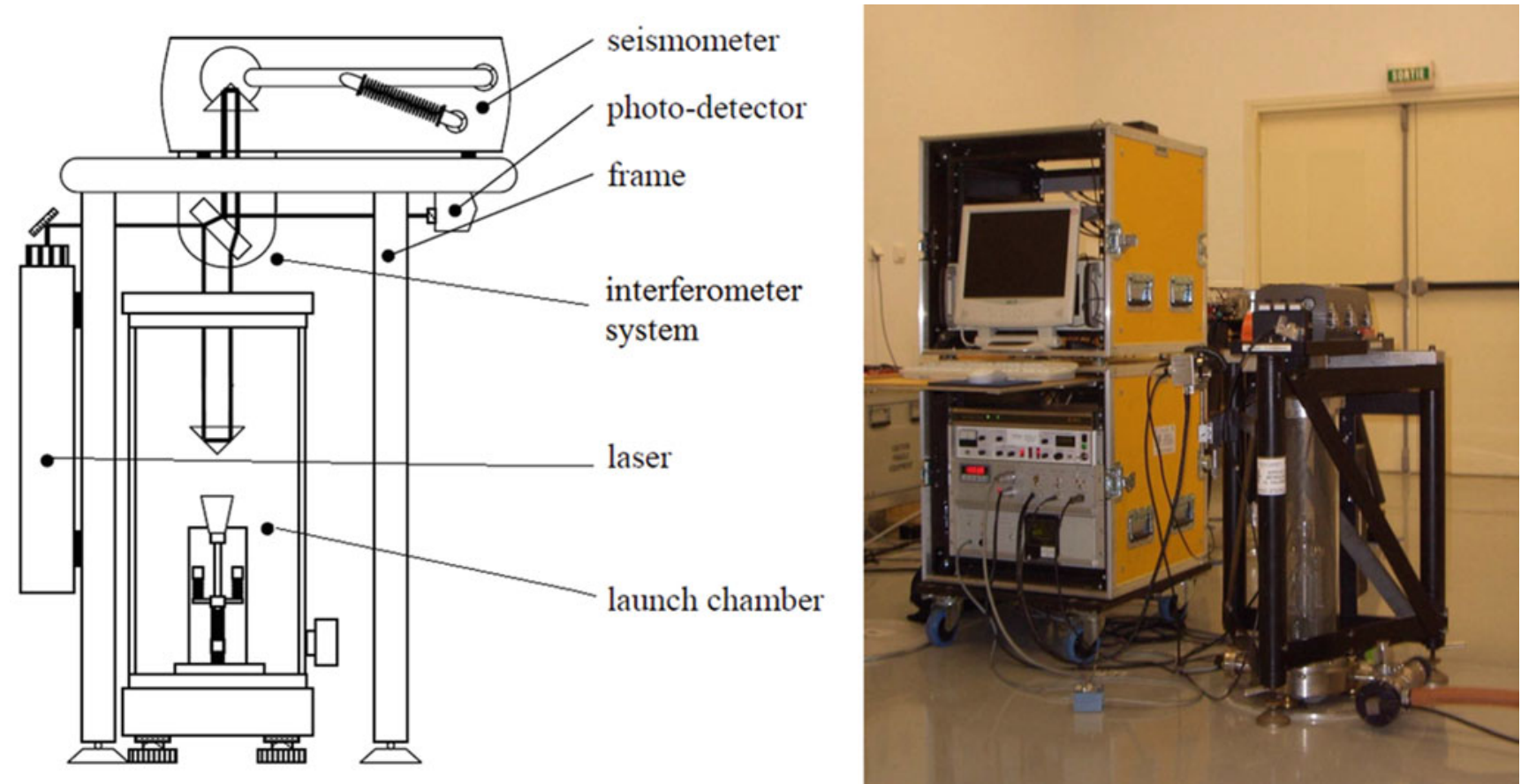

Fig. 1 Schematic drawing and picture of the IMGC-02 absolute gravimeter

cube retroreflector and a second retroreflector mounted on the quasi-inertial mass of a vibration isolation system. The gravimeter is composed of five main parts: (1) a launch system in a vacuum cylinder, (2) an interferometer system connected to an anti-vibrating system, (3) a laser body, (4) a photodetector and (5) a supporting frame, as shown in Fig. 1. A detailed description of the gravimeter can be found in D'Agostino (2006) and D'Agostino et al. (2008). However, the current launch system, which is composed of a moveable carriage supported by two pairs of springs, and the interferometer system, which is a modified MachZehnder interferometer, introduce uncertainty contributions and practical problems that need to be overcome. For this reason, new launch and interferometer systems have been designed. This work deals with the description of the current systems and their future improvements.

\section{2}

\section{The Launch System}

The current launch system is composed of the vacuum chamber, the test mass and the launching pad. The launch chamber can be approximated to a cylinder with a diameter of $14 \mathrm{~cm}$ and a height of $65 \mathrm{~cm}$. The base of the vacuum chamber is made of stainless steel and is supported by three legs equipped with levelling screws, which allow the vertical alignment of the chamber. The main part of the vacuum chamber is a flanged glass pipe, whose bottom part is fitted to the base, whereas the upper part is sealed with an aluminium cover. A BK7 glass window $(5 \mathrm{~cm}$ diameter, $1.27 \mathrm{~cm}$ thick, $\lambda / 10$ flatness, parallelism better than 2 arcsec) is positioned at the centre of this cover and allows the laser beam to reach the test mass. Connections are sealed by O-rings and, inside the glass pipe, a Faraday cage shields the test mass from electrostatic charges. The base of the chamber has two arm pipes: the first one is connected to a low noise turbo pump, the second to an ionisation vacuum gauge. A rotary pump carries out the coarse evacuation. A pressure of $1 \times 10^{-3} \mathrm{~Pa}$ is reached after about $5 \mathrm{~h}$.

The launching pad (Fig. 2) can be approximated to a $5 \times 5 \times 20 \mathrm{~cm}^{3}$ parallelepiped and is tightly connected to the base of the vacuum chamber. It supports, throws up and catches the test mass at the end of its trajectory. The test mass is a corner cube retroreflector, which has the property to reflect an incident ray parallel to itself regardless of the angular orientation. The corner cube has a mass of $0.15 \mathrm{~kg}$ and lies horizontally on three supports, which are the vertices of an equilateral triangle. These supports constitute the upper ending part of the catcher, which is fixed to a moveable carriage $(0.15 \mathrm{~kg})$ on a vertical linear bearing rail, screwed to an aluminium rectangular support. The carriage, in turn, is fixed to two pairs of series springs and is retained by an electromagnet. The starting horizontal position of the test mass, resting on the catcher, corresponds to the best alignment of the interferometer, i.e. the best overlapping between the test and reference beams. The thrust of the system (around $27 \mathrm{~N}$ ) is given by the two pairs of series springs (elastic modulus approximately $1.1 \mathrm{~N} \mathrm{~mm}^{-1}$ each), 

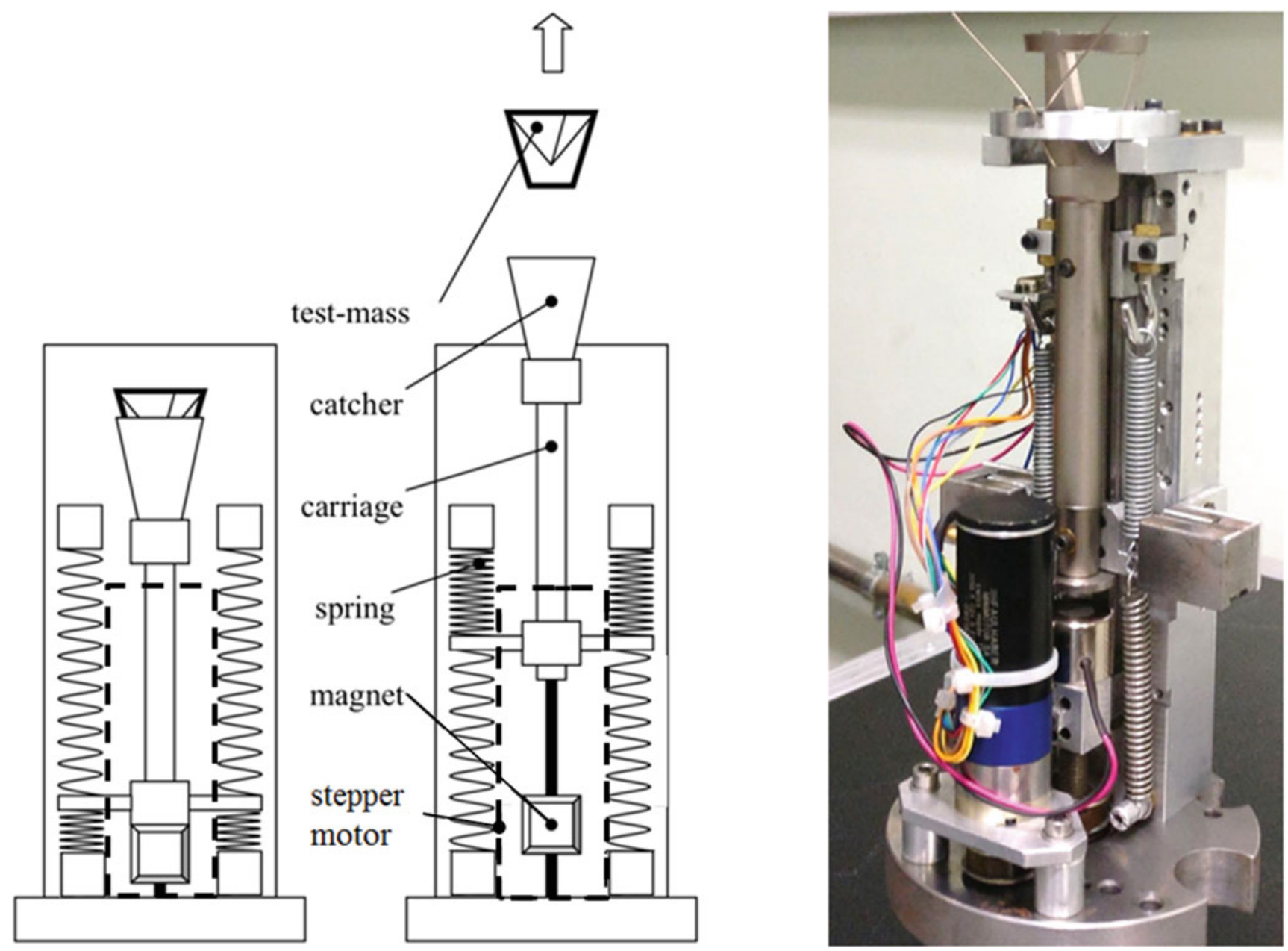

Fig. 2 Schematic drawing (left) and picture (right) of the current launching pad

which are arranged in parallel and loaded through a screw gear driven by an electric stepper motor. The vertical motion of the test mass is triggered by cutting off the exciting current of the magnet. The impulse is transmitted through the three supporting points to the test mass. When the mechanical action ends, the body hovers and its trajectory is tracked. The resulting total stroke of the mechanical system, before the release of the test mass, is around $25 \mathrm{~mm}$. When released, the test mass has a speed of about $2 \mathrm{~m} \mathrm{~s}^{-1}$ and travels in the vacuum chamber a distance of about $20 \mathrm{~cm}$. The launch system is automatically reloaded after the pad catches the free-falling test mass.

Ideally, the corner cube retroreflector realizes a point in space. Unfortunately, the corner cube's centre of mass is not coincident with its optical centre. For this reason, the corner cube is fitted in an aluminium frame, designed in such a way as to move the centre of mass of the assembly as close as possible to the optical centre. In this way, any rotation of the test mass around its centre of mass should affect neither the interferometer alignment nor the measured $g$ value. Despite the highest accuracy in the realization of the support, a small but unavoidable difference between the optical centre and the centre of mass is still present. This entails that beam shear effects and rotational effects might occur due to movements and rotations of the test mass on the horizontal plane, caused by (small) different loadings of the springs which generate, during the upward launch, lateral forces and moments on the moveable carriage. As a consequence, the two interfering beams can translate relative to each other; launches affected by a fringe visibility reduction above a fixed threshold (usually 10-20\% of the maximum intensity) are rejected. The rotational effect, instead, introduces a centripetal acceleration, whose vertical component is added to the local gravity acceleration $g$. Experimental tests (D'Agostino 2006) show that the average rotation of the test mass during the trajectory is $10 \mathrm{mrad}$ and the associated angular velocity is $25 \mathrm{mrad} \mathrm{s}^{-1}$, given a total flying time of around $400 \mathrm{~ms}$. Considering the uncertainty in locating the optical centre and the centre of mass as a random variable with a uniform distribution within $\pm 0.1 \mathrm{~mm}$, the expected uncertainty due to the rotation of the 

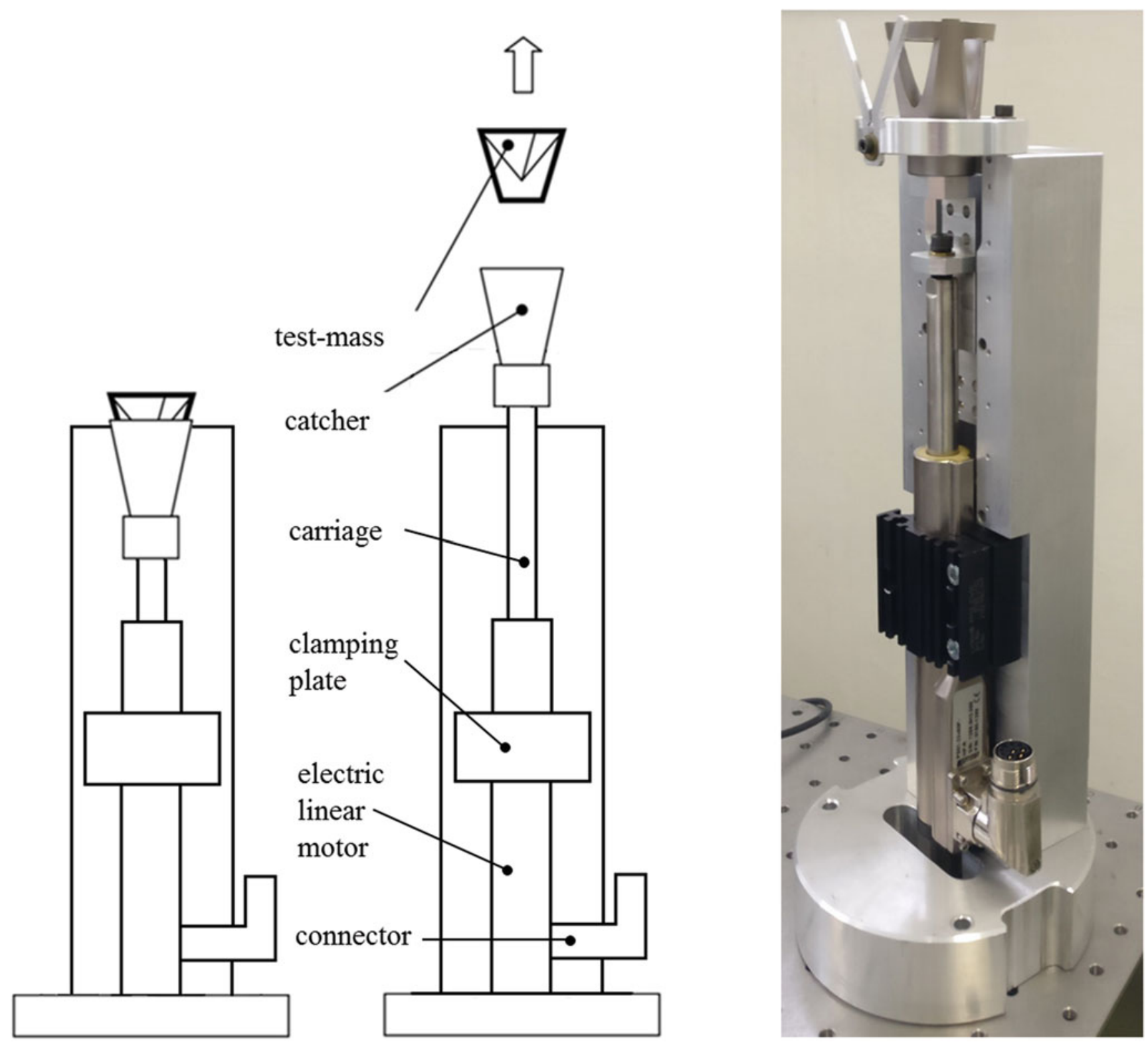

Fig. 3 Schematic drawing (left) and picture (right) of the new launching pad

corner cube is $3.6 \mu \mathrm{Gal}$ and represents one of the largest contribution to the uncertainty budget (D'Agostino et al. 2008).

To overcome these issues, a new launch system has been designed (Fig. 3). It can be approximated to a $7 \times 7 \times 26 \mathrm{~cm}^{3}$ parallelepiped and consists of an electric linear motor, which has its stator and rotor unrolled to produce a linear force along its length, to which the moveable carriage and the catcher are fixed. As in the current system, the moveable carriage slides on a linear bearing rail screwed to an aluminium rectangular support. In this way, once aligned, beam shear and rotational effects should be minimized. A $50 \%$ decrease in the number of rejected launches is foreseen, and the rotational effect uncertainty is expected at least to halve. The linear motor has a maximum stroke of $780 \mathrm{~mm}$, a peak force of $67 \mathrm{~N}$ and a continuous maximum force of $14 \mathrm{~N}$. The presence of motor flanges enables the easy mounting of the linear motor on the aluminium structure, while the clamping plate design enables quick assembly and disassembly of the linear motors without disassembling the flange. The rate of movement of the magnetic field is electronically controlled via software, which allows one to track the motion of the rotor. In this way, it is also possible to design the downward motion profile in order to collect the test mass at the end of its falling motion. 


\section{The Interferometer System}

The current interferometer system is a modified MachZehnder interferometer (Germak et al. 2002; D'Agostino 2006). A scheme is reported in Fig. 4. The light emitted by the He-Ne laser $(\lambda \approx 633 \mathrm{~nm})$ passes through an optical Faraday isolator (FI) which avoids any backreflection into the laser, possibly changing its wavelength and thus affecting the measurement. After the Faraday isolator, the beam is enlarged to $2 \mathrm{~mm}$ spot size by a beam expander (BE) and proceeds vertically toward a beam splitter (BS). The reflected beam proceeds horizontally toward a small corner cube $\left(\mathrm{S}_{\mathrm{CC}}\right)$ used to observe the vertical orientation of the beam through a telescope $(\mathrm{T})$. The transmitted beam, vertically directed, is deviated by a moveable mirror $\left(\mathrm{M}_{1}\right)$ which can be rotated around two axes to adjust the direction of the shifting arm of the interferometer to the true vertical. These elements are needed to check the verticality of the laser beam. Afterwards the beam enters horizontally into the optical prism (OP) situated beneath the reference corner cube retroreflector $\left(R_{R}\right)$. The OP has been designed in such a way that one half is a reflecting mirror and the other half is a beam splitter. The incident light is divided into two beams by OP. One of the beams, which represents the fixed arm of the interferometer, proceeds horizontally and goes straight toward the detector (D); the second beam travels vertically down to the test mass's corner cube retroreflector $\left(T_{R}\right)$ and forms the shifting arm of the interferometer; it is reflected back to $R_{R}$ and strikes the movable mirror $\left(\mathrm{M}_{2}\right)$ after being reflected by the OP. The adjustment of $\mathrm{M}_{2}$ allows the beams to recombine at the second beam splitting portion of OP. The interference fringe causes cyclic variations in intensity, which are due to the change in the difference between the two optical path lengths at every half-wavelength displacement of the test mass retroreflector $T_{R}$. The detector converts the output light of the interferometer to an electric signal. The test mass trajectory is measured by timing this electric signal. Since the recombining beams have to be coaxial in order to avoid distortions on the laser interference fringes, the angular position of the mirror $\mathrm{M}_{2}$ has to be adjusted every time before the measurement and has to be monitored during the measurement. The alignment is achieved by rotating the mirror $\mathrm{M}_{2}$ around its two axes through a piezoelectric tilt actuator.

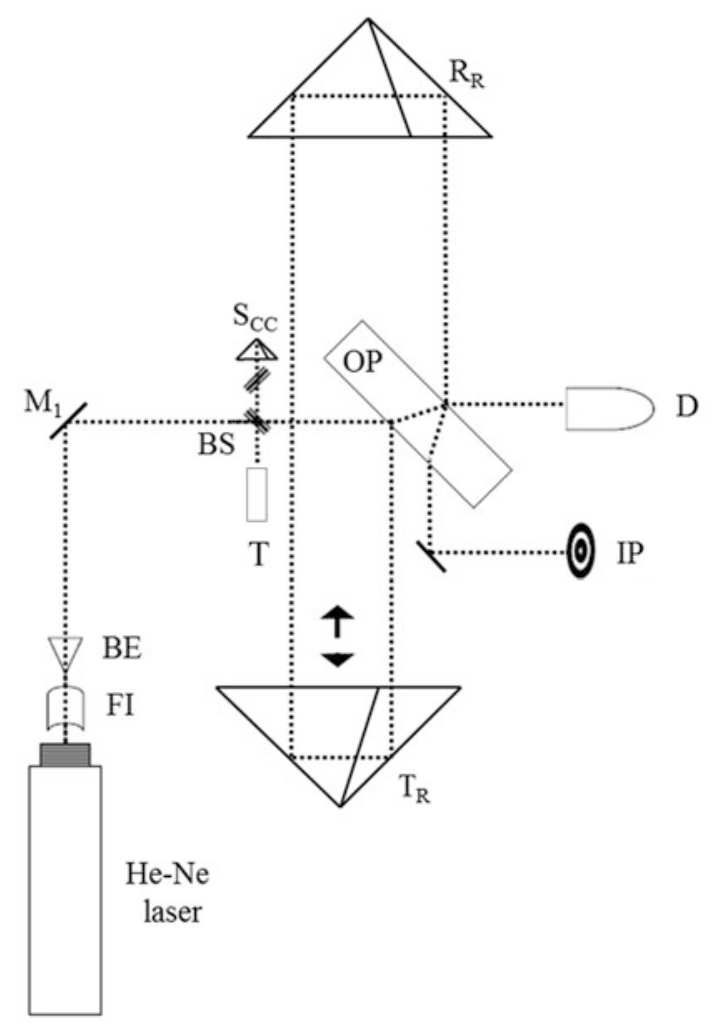

The new modified Jamin interferometer

\section{The modified Mach-Zehnder interferometer}

Fig. 4 Scheme of the current modified Mach-Zehnder interferometer (left) compared to the new modified Jamin interferometer (right). Abbreviations: OP optical prism, BE beam expander, FI Faraday iso- lator, BS beam splitter, D detector, T telescope, IP interference pattern control, $\mathrm{M}$ mirror, $\mathrm{R}_{\mathrm{R}}$ reference retroreflector corner-cube, $\mathrm{T}_{\mathrm{R}}$ test-mass retroreflector corner-cube, $\mathrm{S}_{\mathrm{CC}}$ small corner-cube 


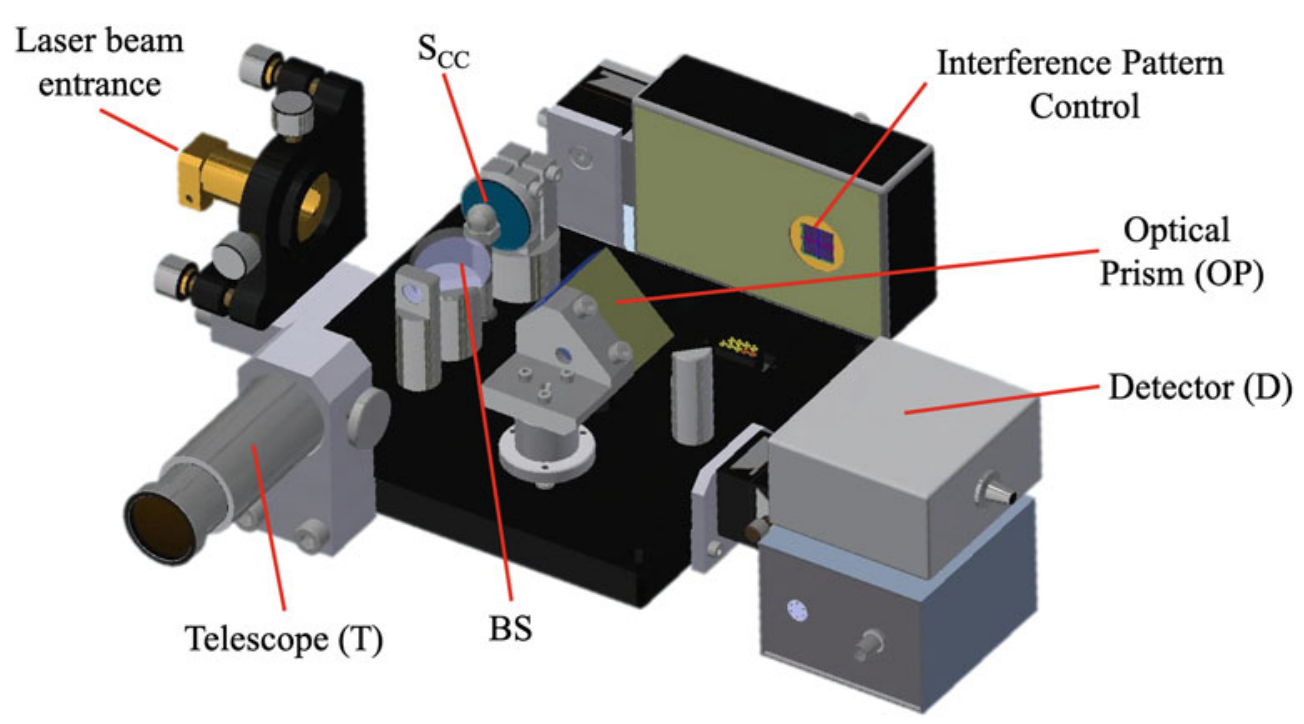

Fig. 5 3D-Scheme of the new modified Jamin interferometer on the horizontal plane

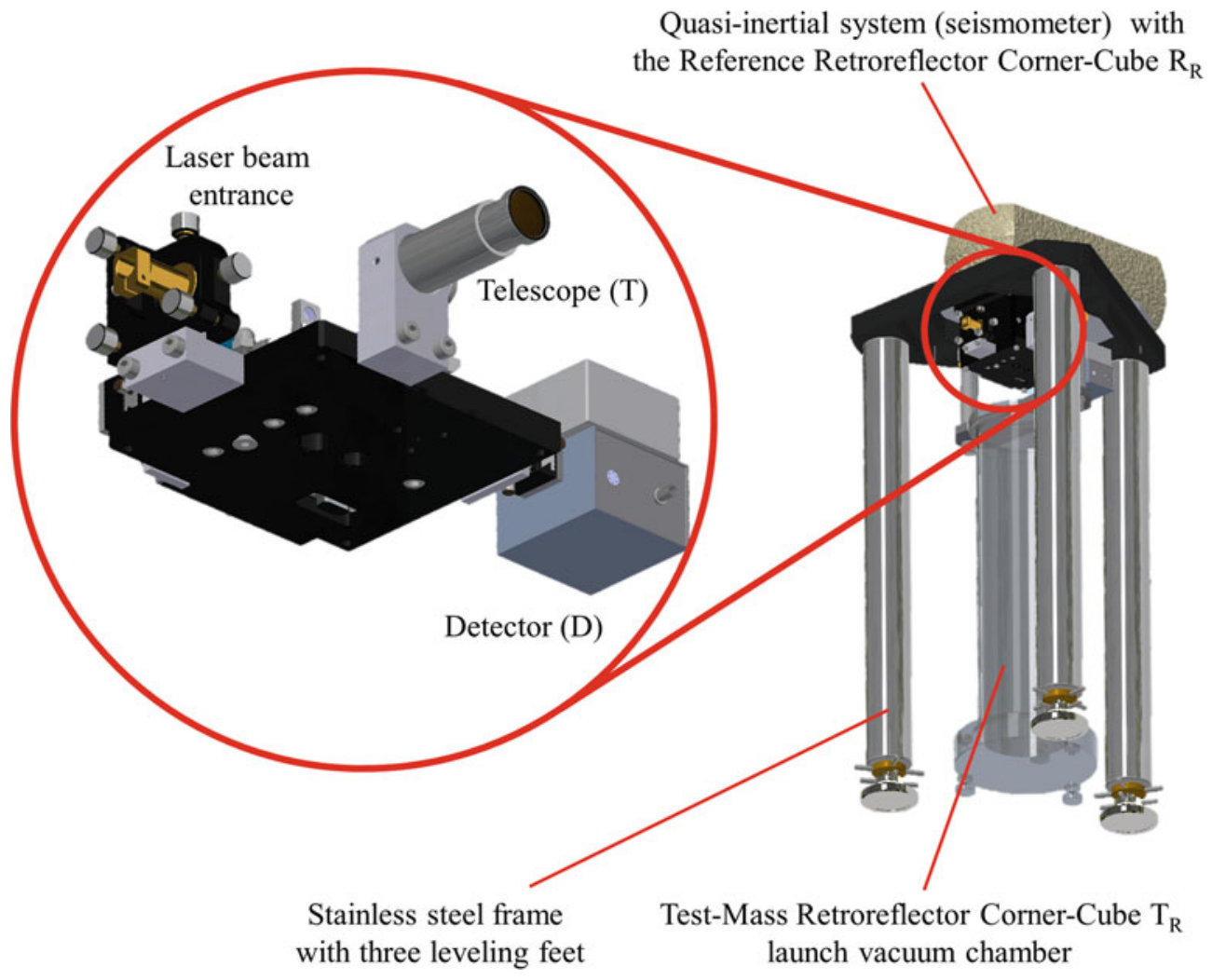

Fig. 6 Bottom view of the 3D-Scheme of the new modified Jamin interferometer integrated in the IMGC-02 rise-and-fall ballistic gravimeter

Unfortunately, this operation entails practical problems, is highly time consuming and has to be performed before and during the measurement session. For this reason, a new modified Jamin interferometer (Shamir 1999) has been devised (Fig. 4). Such system is similar to the modified Mach-Zehnder interferometer except that the two beams directly recombine on the OP, thus the movable mirror $\mathrm{M}_{2}$ is removed. A 3-D detail of the main part of the new system is depicted in Fig. 5, while its assembly in the gravimeter is shown in Fig. 6. The alignment of the recombined beams is possible by just shifting the reference corner cube retroreflector $\mathrm{R}_{\mathrm{R}}$ along the horizontal plane. The main advantages are a simpler and faster alignment of the two beams and a better stability in time entailing an expected setting time reduction 
of around $25 \%$ and a further decrease of rejected throws. The new scheme has a potential Abbe error because of the removal of $\mathrm{M}_{2}$, which was used to correct the misalignment of the beams traveling in the two arms of the interferometer; using high optical quality corner cubes and prism (angular accuracy within 1 arcsec and a flatness within $\lambda / 10$ ) the uncertainty contribution due to the Abbe error is minimized.

\section{$4 \quad$ Conclusions}

For the measurement of the acceleration due to gravity, INRiM developed a transportable ballistic rise-and-fall absolute gravimeter: the IMGC-02. Currently, the launch system is composed of a moveable carriage supported by two pairs of series springs in parallel, which are loaded by moving down the carriage through a screw gear driven by an electric stepper motor. Nevertheless, it is likely that lateral forces arising during the upward launch of the test mass, caused by unavoidable small different loadings of the two springs, make the test mass move on the horizontal plane and rotate. Therefore, the two interfering beams can translate relative to each other, so that beam shear and rotational effects might occur. To overcome these issues, a new launch system has been designed. It consists of an electric linear motor, which has its stator and rotor unrolled to produce a linear force along its length, fixed to the moveable carriage. The rate of movement of the magnetic field is electronically controlled to track the motion of the rotor. In this way, beam shear and rotational effects should be minimized. For what concern the interferometer system, at present a modified Mach-Zehnder interferometer is adopted. Unfortunately, the alignment operation entails practical problems, is highly time-consuming and has to be performed before and, sometimes, during the measurement session. For this reason, a new modified Jamin interferometer has been devised. This system is similar to the modified Mach-Zehnder interferometer except that the two beams directly recombine on the optical prism, thus the movable mirror is removed. The main advantages are a simpler alignment of the two beams and better stability in time. The new interferometer system should guarantee measurements that are more robust and a faster setup of the gravimeter.

\section{References}

D’Agostino G (2006) Development and metrological characterization of a new transportable absolute gravimeter. $\mathrm{PhD}$ dissertation, Politecnico di Torino, Torino

D’Agostino G, Desogus S, Germak A, Origlia C, Quagliotti D, Berrino G, Corrado G, D'Errico V, Ricciardi G (2008) The new IMGC02 transportable absolute gravimeter: measurement apparatus and applications in geophysics and volcanology. Ann Geophys 51(1):3949

Germak A, Desogus S, Origlia C (2002) Interferometer for the IMGC rise-and-fall absolute gravimeter. Metrologia 39(5):471-475

Shamir J (1999) Optical systems and processes. SPIE, Bellingham

Open Access This chapter is licensed under the terms of the Creative Commons Attribution 4.0 International License (http://creativecommons. org/licenses/by/4.0/), which permits use, sharing, adaptation, distribution and reproduction in any medium or format, as long as you give appropriate credit to the original author(s) and the source, provide a link to the Creative Commons licence and indicate if changes were made.

The images or other third party material in this chapter are included in the chapter's Creative Commons licence, unless indicated otherwise in a credit line to the material. If material is not included in the chapter's Creative Commons licence and your intended use is not permitted by statutory regulation or exceeds the permitted use, you will need to obtain permission directly from the copyright holder.

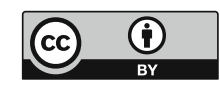

